Acta Crystallographica Section D

Biological

Crystallography

ISSN 0907-4449

Philip M. Leonard, ${ }^{a}$ Caroline M. Marshall, ${ }^{a}$ Eleanor J. Dodson, ${ }^{a}$ Nicholas J. Walton ${ }^{b}$ and Gideon Grogan $^{\mathrm{a} *}$

${ }^{\text {a }}$ ork Structural Biology Laboratory, Department of Chemistry, University of York, Heslington, York YO10 5YW, England, and ${ }^{\mathbf{b}}$ Institute of Food Research, Norwich Research Park, Colney, Norwich NR4 7UA, England

Correspondence e-mail: gg12@york.ac.uk

\title{
Purification, crystallization and preliminary X-ray crystallographic analysis of hydroxycinnamoyl- coenzyme A hydratase-lyase (HCHL), a crotonase homologue active in phenylpropanoid metabolism
}

4-Hydroxycinnamoyl-coenzyme A hydratase-lyase (HCHL), also called feruloyl-CoA hydratase-lyase (FCHL), from Pseudomonas fluorescens strain AN103 is an enzyme of the crotonase superfamily that catalyses the one-step conversion of the CoA thioesters of 4-coumaric acid, caffeic acid and ferulic acid to the aromatic aldehydes 4-hydroxybenzaldehyde, protocatechuic aldehyde and vanillin, respectively. The reaction occurs via a hydration followed by a carbon-carbon bond-cleavage reaction. HCHL has been crystallized by the hanging-drop method of vapour diffusion using polyethylene glycol $20000 \mathrm{Da}$ as the precipitant. The crystals belong to the orthorhombic system, with proposed space group $P 22_{1} 2{ }_{1} 2$ and unit-cell parameters $a=154.2, b=167.5, c=130.8 \AA$. The $V_{\mathrm{M}}$ suggests that the asymmetric unit contains four trimers. Single-wavelength data collection has been undertaken and structure determination is under way by molecular replacement using data collected to $1.8 \AA$ resolution. Determination of the structure of $\mathrm{HCHL}$ will provide insight into the catalytic mechanism of an unusual enzymatic reaction with relevance to the applications of the enzyme in metabolic engineering.

\section{Introduction}

4-Hydroxy-trans-cinnamic acids and their coenzyme A ( CoA) thioesters are of major importance in plant metabolism as precursors of lignin, cell-wall phenolic components and defensive compounds (Dixon \& Paiva, 1995). Ferulic acid (4-hydroxy-3-methoxy-transcinnamic acid) in particular is a widespread component of plant cell walls and its catabolism is essential to the overall biodegradation of plant-derived wastes.

Until comparatively recently, no biochemical mechanism for the degradation of cinnamic acids had been elucidated. The first biochemical and molecular-genetic characterization was reported from Pseudomonas fluorescens strain AN103, an organism selected for growth on ferulic acid as the sole carbon source. P. fluorescens AN103 was shown to degrade ferulic acid (1) via the pathway shown in Fig. 1, wherein vanillin occurs as an intermediate (Gasson et al., 1998). (1) is first coupled to coenzyme A in an ATP-dependent reaction to form feruloyl-CoA (2). (2) is then hydrated to yield the intermediate 4-hydroxy3 -methoxyphenyl- $\beta$-hydroxypropionyl-CoA (HMPHP-CoA) (3), which subsequently undergoes $\mathrm{C}-\mathrm{C}$ bond cleavage to yield the aldehyde vanillin (4) and acetyl-CoA. Both hydration and $\mathrm{C}-\mathrm{C}$ bond-cleavage reactions were determined to be catalysed by the enzyme 4-hydroxycinnamoyl-coenzyme A
Received 13 August 2004

Accepted 30 September 2004
C) 2004 International Union of Crystallography Printed in Denmark - all rights reserved hydratase-lyase (HCHL). The gene encoding HCHL was cloned and expressed and the HCHL protein was characterized biochemically (Mitra et al., 1999), revealing it to be a homologue of the crotonase superfamily, an interesting group of low-sequence-similarity enzymes that are structurally homologous but catalyse a diverse range of chemical reactions (Holden et al., 2001). The HCHL enzyme has a monomer molecular weight of $31007 \mathrm{Da}$, with 273 amino acids per monomer. The two-step hydration/C $-\mathrm{C}$ bond cleavage catalysed by $\mathrm{HCHL}$ is a new addition to the range of reactions catalysed by the crotonase superfamily. Homologous genes encoding HCHL have more recently been reported from Pseudomonas spp. (Overhage et al., 1999; Plaggenborg et al., 2003), from Amycolatopsis sp. (Achterholt et al., 2000) and (at much lower homology) from Delftia acidovorans (Plaggenborg et al., 2001).

HCHL has been expressed heterologously in plants, where it causes a major diversion of the phenylpropanoid pathway and the formation of new products in large amounts, principally glucose conjugates of 4-hydroxybenzoic acid, a monomer for the production of liquid-crystal polymers (Mayer et al., 2001; Mitra et al., 2002; McQualter et al., 2004). These are presumably formed from 4-hydroxybenzaldehyde produced by the action of HCHL on endogenous 4-coumaroyl-CoA. On the other hand, there was no detectable accumulation of the aroma 
<smiles>COc1cc(/C=C/C(=O)O)ccc1O</smiles>

Ferulic acid (1)<smiles>COc1cc(C=O)ccc1O</smiles>

Vanillin (4)

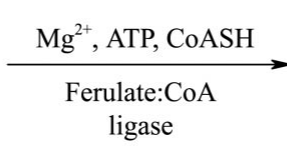



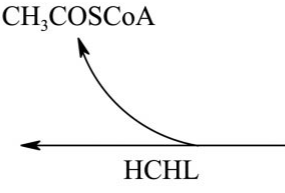<smiles>COc1cc(/C=C/C(=O)O[Na])ccc1O</smiles>

Feruloyl-CoA (2)<smiles>[Y]C(C)C</smiles>

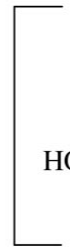

4-Hydroxy-3-methoxyphenyl$\beta$-hydroxypropionyl SCoA (HMPHP-CoA) (3)

Figure 1

Metabolism of ferulic acid by $P$. fluorescens AN103. HCHL, hydroxycinnamoyl-coenzyme A hydratase-lyase.

compound vanillin even in the form of its glucoside.

Our interest in both the structural and mechanistic basis of $\mathrm{C}-\mathrm{C}$ bond cleavage by crotonase homologues (Leonard \& Grogan, 2004) and its applications in metabolic engineering prompted us to begin a study of the structure of HCHL.

\section{Overexpression and purification}

HCHL protein was purified from an overexpressing Escherichia coli strain BL21(DE3) which had been transformed with the plasmid pFI1039 encoding the gene for P. fluorescens biovar V strain AN103 HCHL (Gasson et al., 1998), which had been constructed from the pSP72 plasmid (Novagen). Cells were grown in 4.51 LuriaBertani broth with $100 \mu \mathrm{g} \mathrm{ml}^{-1}$ ampicillin in a rotary shaker at $310 \mathrm{~K}$ until an $\mathrm{OD}_{600}$ of 0.5 was reached. HCHL expression was induced by the addition of $1 \mathrm{~m} M$ isopropyl$\beta$-D-thiogalactopyranoside (IPTG) and cells were allowed to grow for a further $3.5 \mathrm{~h}$. Induced cells were harvested by centrifugation and the cell pellet was resuspended in $30 \mathrm{ml} 50 \mathrm{~m} M$ Tris- $\mathrm{HCl}$ pH 7.1, $1 \mathrm{~m} M$ dithiothreitol (DTT) and $20 \mu M$ phenylmethylsulfonyl fluoride (PMSF) (buffer $A$ ) and disrupted by sonication. The homogenate was then centrifuged for $20 \mathrm{~min}$ at $12000 \mathrm{~g}$, after which a $20-80 \%(w / v)$ ammonium sulfate cut was derived. The protein solution was taken to a concentration of $2 \mathrm{M}$ ammonium sulfate and loaded onto a HiLoad 26/10 phenyl Sepharose column
(Amersham), which was eluted with a decreasing ammonium sulfate concentration. Fractions were analysed by SDSPAGE and those containing HCHL were pooled, concentrated and dialysed against 21 buffer $A$. The protein solution was loaded onto a Mono Q anion-exchange column (Amersham) and eluted against a sodium chloride gradient $(0-0.4 M)$. Fractions were analysed by SDS-PAGE and those containing HCHL were pooled, concentrated and loaded onto a HiLoad 16/60 Superdex 200 prep-grade gel-filtration column (Amersham) equilibrated with buffer $A$. Fractions were analysed by SDSPAGE and those containing HCHL were pooled and concentrated to $10 \mathrm{mg} \mathrm{ml}^{-1}$. The purity of preparation estimated by SDSPAGE was close to $100 \%$.

\section{Crystallization and data collection}

HCHL protein in buffer $A$ was crystallized using the hanging-drop vapour-diffusion technique by mixing $1 \mu \mathrm{l}$ protein solution with an equal volume of precipitant followed by equilibration at $290 \mathrm{~K}$. Crystals were initially grown using the Clear Strategy screen from Molecular Dimensions Ltd (Brzozowski \& Walton, 2001) condition No. 13. After optimization, crystals of maximum dimensions $0.3 \times 0.2 \times 0.05 \mathrm{~mm}$ (Fig. 2) grew within one week at a protein concentration of $10 \mathrm{mg} \mathrm{ml}^{-1}$ in $11 \%(w / v)$ PEG 20000 with $8 \%(v / v)$ PEG 550 monomethyl ether, $0.8 \mathrm{M}$ sodium formate, $0.2 \%(v / v)$
Table 1

Data-collection statistics for HCHL native data.

Values in parentheses refer to the highest resolution shell.

\begin{tabular}{|c|c|}
\hline Beamline & ID14-EH1 \\
\hline Wavelength $(\AA)$ & 0.934 \\
\hline Resolution (§) & $30-1.8(1.85-1.8)$ \\
\hline Unique reflections & 308927 (25526) \\
\hline Completeness (\%) & $100(100)$ \\
\hline$R_{\text {sym }} \dagger(\%)$ & $4.5(33.2)$ \\
\hline Multiplicity & $4.2(4.2)$ \\
\hline$\langle I\rangle / \sigma(I)$ & $21.9(3.3)$ \\
\hline
\end{tabular}

$\dagger R_{\text {sym }}=\sum_{h k l} \sum_{i}\left|I_{i}-\langle I\rangle\right| / \sum_{h k l} \sum_{i}\langle I\rangle$, where $I_{i}$ is the $i$ th measurement and $\langle I\rangle$ is the weighted mean of all measurements of $I$. $\langle I\rangle / \sigma(I)$ indicates the average of the intensity divided by its average standard deviation.

butane-1,4-diol in $0.05 M$ 2-( $N$-morpholino)ethanesulfonic acid buffer $\mathrm{pH}$ 5.6.

A crystal of HCHL was flash-cooled prior to data collection by transferring it into a solution identical to the precipitant before positioning it in a cryostream (Oxford Cryosystems) at $120 \mathrm{~K}$. The crystal was transferred to a liquid-nitrogen storage dewar and transported to the European Synchrotron Radiation Facility (ESRF), where data were collected on station ID14-EH1 at a single wavelength.

\section{Data analysis}

The HCHL data set was autoindexed using the program DENZO (Otwinowski \& Minor, 1997), indicating that the crystals have a primitive orthorhombic lattice with unit-cell parameters $a=130.8, b=154.2$, $c=167.5 \AA$ and therefore a unit-cell volume of $3.38 \times 10^{6} \AA^{3}$. The data were processed and scaled using the $H K L$ suite of programs (Table 1).

Crotonase-family enzymes are known to form trimeric disks with tight interactions between the three subunits (Mursula et al., 2004); of those of known crystal structure, many form a hexamer with 32 symmetry in the crystal lattice through trimer dimerization. In many cases, all or part of the

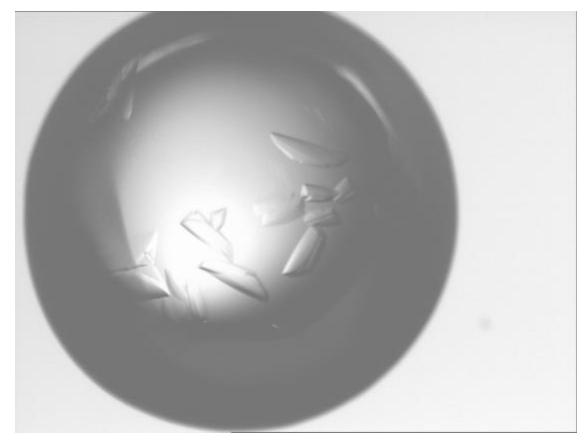

Figure 2

Crystals of hydroxycinnamoyl-coenzyme A hydratase-lyase. 
hexamer is generated by crystal symmetry. For example, the structures of methylmalonyl-CoA decarboxylase (Benning et al., 2000), dienoyl-CoA isomerase (Modis et al., 1998) and 4-chlorobenzoyl-CoA dehalogenase (Benning et al., 1996) all contain a trimer in the asymmetric unit and the structure of $\Delta^{3}-\Delta^{2}$-enoyl CoA isomerase (Mursula et al., 2001) contains a monomer in the asymmetric unit. In this orthorhombic space group the threefold axis and at least two of the three twofold axes must be noncrystallographic. The molecular weight of an isolated molecule is $31007 \mathrm{Da}$ and that of the hexamer is $186042 \mathrm{Da}$. The $V_{\mathrm{M}}$ is $2.3 \AA^{3} \mathrm{Da}^{-1}$ for 12 molecules in the asymmetric unit. The native Patterson map calculated at $4 \AA$ resolution contains a peak at $u=0.34, v=0.3$ and $w=0.5$ which is $25 \%$ of the origin peak height, indicating that there is a strong non-crystallographic translation. It is therefore possible that the asymmetric unit contains four trimers, where we would expect two hexamers in each asymmetric unit or four independent trimers each with their partner trimer generated by the space-group twofold axis.

We would expect the self-rotation function to show a threefold rotation axis perpendicular to three twofold axes, with the twofold axes at $120^{\circ}$ to each other. The self-rotation function calculated using POLARRFN (Collaborative Computational Project, Number 4, 1994) has a peak with $40 \%$ of the origin-peak height in the $\kappa=120^{\circ}$ section at $\omega=90.0, \varphi=63.8, \kappa=119.5^{\circ}$, indicating a threefold axis. The axis has direction cosines $(0.4411,0.8974,0.0000)$. Two symmetry equivalents of the same peak are twofold axes with $\omega=120.2, \varphi=333.8$, $\kappa=180.0^{\circ}$ and $\omega=120.2, \varphi=153.8, \kappa=180.0^{\circ}$. The direction cosines of these twofold axes are $(0.7753,-0.3811,-0.5037)$ and
$(-0.7753,0.3811,-0.5037)$, respectively. Along with the crystallographic twofold axis along $c$ with direction cosines $(0.0,0.0,1.0)$, these form the required set.

The diffraction pattern was inspected using HKLVIEW (Collaborative Computational Project, Number 4, 1994), revealing that those reflections along $0 k 0$ for which $k$ was odd and those along $00 l$ for which $l$ was odd were systematically absent, indicating possible twofold screw axes along $k$ and $l$. This was not the case along $h 00$, indicating that the most likely space group was $P 2_{1} 2_{1} 2$. For this reason $a, b$ and $c$ were reindexed to give revised unit-cell parameters $a=154.2$, $b=167.5, c=130.8 \AA$.

The structure of HCHL is currently being sought by molecular replacement using the structure of rat enoyl-CoA hydratase as a search model. The resulting model of HCHL will provide further information about the diverse reactions performed by members of the crotonase superfamily. The applications of the enzyme in metabolic engineering make it an exciting target for structural and functional analysis.

We would like to thank the Biotechnology and Biological Sciences Research Council (BBSRC) UK for funding. In addition, we would like to thank the Innovation, Priming and Research Fund, University of York for an award to GG. We would also like to thank the staff of the European Synchrotron Radiation Facility (ESRF) for provision of data-collection facilities and Drs John Payne and Arjan Narbad for producing plasmid pFI1039.

\section{References}

Achterholt, S., Priefert, H. \& Steinbüchel, A. (2000). Appl. Microbiol. Biotechnol. 54, 799807.
Benning, M. M., Haller, T., Gerlt, J. A. \& Holden, H. M. (2000). Biochemistry, 38, 4630-4639.

Benning, M. M., Taylor, K. L., Liu, R. Q., Yang, G., Xiang, H., Wesenberg, G., Dunaway-Mariano, D. \& Holden, H. M. (1996). Biochemistry, 35 , 8103-8109.

Brzozowski, A. M. \& Walton, J. (2001). J. Appl. Cryst. 34, 97-101.

Collaborative Computational Project, Number 4 (1994). Acta Cryst. D50, 760-763.

Dixon, R. A. \& Paiva, N. L. (1995). Plant Cell, 7, 1085-1097.

Gasson, M. J., Kitamura, Y., McLauchlan, W. R., Narbad, A., Parr, A. J., Parsons, E. L. H., Payne, J., Rhodes, M. J. C. \& Walton, N. J. (1998). J. Biol. Chem. 273, 4163-4170.

Holden, H. M., Benning, M. M., Haller, T. \& Gerlt, J. A. (2001). Acc. Chem. Res. 34, 145-157.

Leonard, P. M. \& Grogan, G. (2004). J. Biol. Chem. 279, 31312-31317.

McQualter, R. B., Fong Chong, B., Meyer, K., Van Dyk, D. E., O'Shea, M. G., Walton, N. J., Viitanen, P. V. \& Brumbley, S. M. (2004). In the press.

Mayer, M., Narbad, A., Parr, A. J., Parker, M. L., Walton, N., Mellon, F. A. \& Michael, A. J. (2001). Plant Cell, 13, 1669-1682.

Mitra, A., Kitamura, Y., Gasson, M. J., Narbad, A., Parr, A. J., Payne, J., Rhodes, M. J. C., Sewter, C. \& Walton, N. J. (1999). Arch. Biochem. Biophys. 365, 10-16.

Mitra, A., Mayer, M. J., Mellon, F. A., Michael, A. J., Narbad, A., Parr, A. J., Waldron, K. W. \& Walton, N. J. (2002). Planta, 215, 79-89.

Modis, Y., Filppula, S. A., Novikov, D. K., Norledge, B., Hiltunen, J. K. \& Wierenga, R. K. (1998). Structure, 6, 957-970.

Mursula, A. M., Hiltunen, J. K. \& Wierenga, R. K. (2004). FEBS Lett. 557, 81-87.

Mursula, A. M., van Aalten, D. M. F., Hiltunen, J. K. \& Wierenga, R. K. (2001). J. Mol. Biol. 309, $845-853$.

Otwinowski, Z. \& Minor, W. (1997). Methods Enzymol. 276, 307-326.

Overhage, J., Priefert, H., Rabenhorst, J. \& Steinbüchel, A. (1999). Appl. Environ. Microbiol. 65, 4837-4847.

Plaggenborg, R., Overhage, J., Steinbüchel, A. \& Priefert, H. (2003). Appl. Microbiol. Biotechnol. 61, 528-535.

Plaggenborg, R., Steinbüchel, A. \& Priefert, H. (2001). FEMS Microbiol. Lett. 205, 9-16. 